\title{
Risk Factors and Evolution of Ventilator-Associated Pneumonia by Staphylococcus aureus Sensitive or Resistant to Oxacillin in Patients at the Intensive Care Unit of a Brazilian University Hospital
}

\author{
Michel R. Moreira ${ }^{1}$, Renata L. Cardoso ${ }^{1}$, Alair B. Almeida ${ }^{2}$ and Paulo P. Gontijo Filho ${ }^{1}$ \\ ${ }^{1}$ Uberlândia Federal University, Laboratory of Microbiology; ${ }^{2}$ Uberlândia Federal University Hospital Clinic; Uberlândia, MG, Brazil
}

\begin{abstract}
This study investigated the participation and risk factors of VAP by resistant (ORSA) or sensitive (OSSA) S. aureus to oxacillin and evaluated the implications of adequate or inadequate empirical antimicrobial therapeutics in its evolution in patients interned in a mixing ICU of adults. A patient control-case study with PAVs by ORSA and OSSA was carried out from May 2005 to April 2007 involving 993 patients. VAP was defined based on clinical, radiological, and microbiological $\left(\geq 10^{6} \mathrm{CFU} / \mathrm{mL}\right.$ count in the tracheal aspirate) criteria. Four hundred and seventy four (47.7\%) patients were submitted to mechanical ventilation with $141(29.7 \%)$ VAPs, with $S$. aureus as the most frequent agent (41.2\%). The phenotype ORSA accounted for $47.5 \%$ and OSSA for $52.5 \%$, predominant in late-onset VAPs with frequencies of $93.1 \%$ and $68.7 \%$, respectively. Age $>60$, use of corticoid and previous antibiotic therapy were related $(p<0.05)$ with the development of VAP by ORSA. Mortality rate was higher $(p>0.05)$ in the group with VAP by ORSA (37.9\%). S. aureus was the main agent of VAPs, around half by ORSA, associated with age, late-onset VAP development and previous use of antibiotics and corticoids, but with no significant difference in mortality compared with VAP by OSSA.
\end{abstract}

Key-Words: Ventilator-associated pneumonia, hospital infection, Staphylococcus aureus, resistant microorganisms.

Ventilator-associated pneumonia (VAP) is a hospital infection frequently occurring at the ICU $[1,2]$ and Staphylococcus aureus is one of its most common agents $[2,3]$. Its frequency, as well as proportion of isolates resistant to oxacillin (ORSA) varies among hospitals and ICUs [4]. Increasing hospital infections by oxacillin-resistant Staphylococcus aureus [4,5] led to a greater consumption of vancomycin, the choice antibiotic for hospital infections caused by Gram-positive resistant cocci $[6,7]$.

Due to its gravity, treatment of VAP must be initiated quickly, remaining empirical until the culture and antimicrobial susceptibility test results become available, since VAP may be polymicrobial and can be caused by a wide spectrum of bacterial pathogens, or involve microorganisms, which are multiresistant to antibiotics [8-10].

Until recently, the glycopeptides (teicoplanin and vancomycin) were the only antimicrobials available to treat infections by ORSA $[8,11]$. Although vancomycin is still considered the choice agent to treat infections by ORSA, studies suggest that treatment with vancomycin may vary and may be ineffective, although laboratory data show that the pathogen is susceptible to the antibiotic [8,12]. The problem becomes worse in lung infections due to its unsatisfactory pharmacokynetic profile [12]. Its tissue penetration and concentration in the epithelial lining fluid does not exceed $20 \%$ of the plasma level, with high intra and inter-individual variations [13].

Received on 11 April 2008; revised 18 November 2008.

Address for correspondence: Dr. Michel Rodrigues Moreira. Rua Saturnino Pedro dos Santos 191/104, B. Jardim Finotti, Uberlândia/ MG/Brazil - Zip code: 38408-090. Telephone (0xx34) 9196-2314. Email: moreira.mr@hotmail.com. Finantial: Conselho Nacional de Desenvolvimento Científico e Tecnológico - CNPq.

The Brazilian Journal of Infectious Diseases 2008;12(6):499-503. (C) 2008 by The Brazilian Journal of Infectious Diseases and Contexto Publishing. All rights reserved.
New therapeutic options, but still reduced for ORSA treatment, were developed, such as tigecycline and linezolid [9]. The latter, a synthetic oxazolidinone, presents a good pharmacokinetic profile, with oral bio-availability reaching $100 \%$, allowing sequential therapeutics with oralparenteral transition, after hospital release $[8,9,11]$. In addition to these properties, it is a small molecule that penetrates well in the tissues, including the lung tissues $[9,14]$. Although its use is well-tolerated, it is associated with various side effects [9].

The objective of this work was to investigate the etiology and risk factors of VAP by $S$. aureus resistant or sensitive to oxacillin and to evaluate its evolution when an adequate empirical antimicrobial therapeutics was applied to patients interned in a mixing ICU of adults.

\section{Material and Methods}

Hospital

The Hospital de Clínicas da Universidade Federal de Uberlândia (Uberlândia Federal University Hospital Clinic/HCUFU) is a teaching hospital, with 500 beds, and a mixing ICU of adults with 15 beds.

\section{Study Design}

A control-case study was carried out using patients with VAP by ORSA and OSSA from May 2005 to April 2007, when 993 patients were admitted. The VAPs were defined based on clinical, radiological and microbiological criteria.

\section{Definition of Hospital VAP}

The patients were under mechanical ventilator for a period $\geq 48$ hours after being admitted to the ICU, with new and/or progressive radiological infiltrate and at least under two of the following criteria: purulent sputum, temperature higher than $38.5^{\circ} \mathrm{C}$ or lower than $35^{\circ} \mathrm{C}$, and leukocyte count higher 
than $10,000 / \mu \mathrm{L}$ with deviation to the left or lower than 3,000/ $\mu \mathrm{L}$; and positive quantitative culture of the endotracheal aspirate (count $\geq 10^{6} \mathrm{CFU} / \mathrm{mL}$ ) $[1,2,15]$.

\section{Inadequate Empirical Therapeutics}

Treatment of PAV by ORSA with beta-lactam and by OSSA with vancomycin instead of beta-lactam [8,13].

Additionally, the following patient data were collected: age, gender, invasive procedures, admission diagnosis, previous use of antibiotics and corticoids, hospitalization and mechanical ventilation times. The Ethics Committee for Human Research of the Uberlândia Federal University (UFU) approved the project.

\section{Microbiological Techniques}

Endotracheal aspirate was collected by probe no. 12 early in the morning by health professionals in charge of the procedure (physiotherapists and nurses), and transported in a sterile tube to the Microbiology Laboratory of the UFU. Quantitative culture was carried out in salty Manitol agar (Biobrás, Brazil), MacConckey agar (Difco, France) and Pseudomonas agar (Difco, France). The Staphylococcus aureus samples were identified by manitol fermentation, Gram stain, and catalase, free coagulase and bond coagulase tests. Resistance to oxacillin was detected by means of a screening test in Mueller Hinton agar (Micromed, Brazil) incorporated with $6 \mu \mathrm{g} / \mathrm{mL}$ of oxacillin and $4.5 \%$ of $\mathrm{NaCl}$. The Pseudomonas aeruginosa and the other Gramnegatives were identified by classic techniques routinely applied by the UFU Microbiology Laboratory.

\section{Statistical Analysis}

Univariant comparisons were carried out by the chi-square $\left(\chi^{2}\right)$ and Fisher's Exact tests and the results were considered statistically significant at level of 5\%. The epidemiological data were analyzed through the program Statística 4.5 for Windows and Epi-Info version 5.0 [16].

\section{Results}

A total of 993 patients were admitted to the adult ICU of the HC-UFU. 474 (47.7\%) patients were submitted to mechanical ventilation, with 141 (29.7\%) VAPs, with S. aureus being the most frequent agent (41.2\%) (Figure1). The phenotype ORSA accounted for $47.5 \%$ and OSSA, 52.5\% (Figure 2), predominant in late-onset VAPs with frequencies of $93.1 \%$ and $68.7 \%$, respectively (Table 1 ).

Age $>60$, use of corticoids and previous antibiotic therapy $(p<0.05)$ were related with the development of VAPs by ORSA (Table 1). Inadequate antibiotic therapy in this group was $37.9 \%$, lower $(\mathrm{p}>0.05)$ than that observed in the cases of VAP by OSSA. Mortality rate was higher ( $>0.05$ ) in the group with VAP by ORSA, $37.9 \%$ vs. $25 \%$ in the group with OSSA, but with no statistical significance (Tables 1 and 2 ).

\section{Discussion}

S. aureus was the main agent of the VAPs (41.2\%) among adult patients at the ICU of the HC-UFU, with approximately half (47.5\%) the episodes being caused by the ORSA samples. Age $>60$, use of corticoid and previous antibiotic therapy were the risk factors associated with development of VAPs by ORSA. These factors correspond to those reported in the literature, with the following also predominating: chronic obstructive pulmonary disease and prolonged time of mechanical ventilation $[3,9,15,17,18]$. The VAPs by OSSA occur more frequently in younger patients with traumatic brain injury $[1,3,17]$, as confirmed in our study, with $46.9 \%$ vs. $31.0 \%$ in those infected by ORSA.

The proportion of $S$. aureus samples resistant to oxacillin in the USA is approximately $50 \%$ in critical patients [19,20]. In Brazil, data vary with studies conducted in Rio Grande do Sul and Paraná reporting a participation of $86.7 \%$ and $42,0 \%$ of ORSA, respectively $[21,22]$. In our work, this proportion was closer to the American data.

Resistance to beta-lactams is located on the staphylococcal cassette chromosome (SCCmec), a mobile genetic element, and is encoded by a gene known as mecA, which is regulated by two other genes called mec1 and mecR1. The resistance results of a penicillin-binding protein (PBP2') with lower affinity by this antibiotics. Besides the resistance to beta-lactams, the SCCmec includes transposons and plasmids with genes associated to resistance to others antimicrobial agents like: aminoglycosides, macrolides, quinolones, tetracyclines and clindamicin [23,24].

Currently, three clinically relevant types of ORSA strain exist. The original ORSA that emerged in hospitals in the 1970s is referred to as hospital-acquired ORSA (HA-ORSA). Over the past few decades, HA-ORSA strains made their way into the community. HA-ORSA from the community later admitted to hospitals are referred to as community-onset ORSA (COORSA). For these reasons, CO-ORSA strains have the same clinical features/virulence, and are effectively treated as HAORSA strains. The latest recognized strain type of ORSA is community-acquired ORSA (CA-ORSA), that is exceptionally virulent when have the Panton-Valentine leucocidin and is sensitive to antibiotics such clindamycin, cotrimoxazole and doxycycline, which are not tradicionally active against HAORSA [25].

Measures to prevent the development of multidrugresistant microorganisms, specially the ORSA VAP are composed of general VAP prevention strategies, like basic hygiene $[19,26]$. Appropriate antimicrobial stewardship that includes optimal selection, dose, and duration of treatment, as well as control of antibiotic use, will prevent or slow the emergence of resistance among the microorganisms $[27,28]$.

Early and late-onset VAPs in the HC-UFU were related respectively, to OSSA (83.3\%) and ORSA(55.1\%), as reported by other authors $[2,8,29]$. Late-onset VAPs are associated with predisposing factors such as hospitalization time, use of mechanical ventilator and previous use of antimicrobials, 
Table 1. Characteristics of ventilator-associated pneumonia (VAP) by S. aureus with resistance (ORSA) or sensitivity (OSSA) to oxacillin in interned patients of the ICU for adult patients of the Uberlândia Federal University Hospital Clinic (HC-UFU), from May 2005 to April 2007.

\begin{tabular}{|c|c|c|c|c|c|}
\hline \multirow[b]{2}{*}{ Characteristics } & ORSA & OSSA & \multirow[b]{2}{*}{$\mathbf{P}$} & \multirow[b]{2}{*}{ OR } & \multirow[b]{2}{*}{ CI } \\
\hline & $\mathrm{N}=29(\%)$ & $\mathrm{N}=32(\%)$ & & & \\
\hline \multicolumn{6}{|l|}{ Gender } \\
\hline Male & $21(72.4 \%)$ & 25 (78.1\%) & 0.83 & 0.73 & $0.20-2.73$ \\
\hline Female & $8(27.6 \%)$ & 7 (21.9\%) & 0.82 & 1.36 & $0.37-5.10$ \\
\hline Age $>60$ & $16(55.2 \%)$ & $6(18.8 \%)$ & $0.007^{\#}$ & 5.33 & $1.48-20.04$ \\
\hline Hospitalization time $>8$ days & $6(89.7 \%)$ & $29(90.6 \%)$ & 1.00 & 0.90 & $0.13-6.25$ \\
\hline $\mathrm{VM}^{\mathrm{A}}$ Time $>7$ days & $25(86.2 \%)$ & $28(87.5 \%)$ & 1.00 & 0.89 & $0.16-4.88$ \\
\hline \multicolumn{6}{|l|}{ Invasive procedures } \\
\hline Tracheostomy & $18(62.1 \%)$ & 11 (34.4\%) & 0.06 & 3.12 & $0.97-10.26$ \\
\hline $\mathrm{CVC}^{+}$ & 28 (96.5\%) & 31 (96.9\%) & 1.00 & 0.90 & $0.02-35.02$ \\
\hline $\mathrm{SV}^{++}$ & $28(96.5 \%)$ & 31 (96.9\%) & 1.00 & 0.90 & $0.02-35.02$ \\
\hline $\mathrm{SNG}^{+++}$ & 22 (75.9\%) & $20(62.5 \%)$ & 0.39 & 1.89 & $0.55-6.65$ \\
\hline Early-onset VAP & 2 (6.9\%) & $10(31.3 \%)$ & $0.04^{\#}$ & 0.16 & $0.02-0.93$ \\
\hline Late-onset VAP & 27 (93.1\%) & $22(68.7 \%)$ & $0.04^{\#}$ & 6.14 & $1.07-45.66$ \\
\hline \multicolumn{6}{|l|}{ Etiology } \\
\hline Single microbials & $24(82.8 \%)$ & $20(62.5 \%)$ & 0.14 & 2.88 & $0.76-11.42$ \\
\hline Mixed & $5(17.2 \%)$ & 12 (37.5\%) & 0.14 & 0.35 & $0.09-1.32$ \\
\hline Use of corticoids & 15 (51.7\%) & $5(15.6 \%)$ & $0.006^{\#}$ & 5.79 & $1.53-23.18$ \\
\hline Previous antibiotic therapy & 26 (89.7\%) & 19 (59.4\%) & $0.02^{\#}$ & 5.93 & $1.30-30.74$ \\
\hline \multicolumn{6}{|l|}{ Admission diagnosis } \\
\hline Clinical & $13(44.8 \%)$ & 12 (37.5\%) & 0.75 & 1.35 & $0.43-4.28$ \\
\hline Surgical & 7 (24.1\%) & $5(15.6 \%)$ & 0.60 & 1.72 & $0.41-7.40$ \\
\hline Trauma & 9 (31.0\%) & $15(46.9 \%)$ & 0.32 & 0.51 & $0.16-1.64$ \\
\hline Mortality & $11(37.9 \%)$ & $8(25.0 \%)$ & 0.41 & 1.83 & $0.54-6.34$ \\
\hline \multicolumn{6}{|l|}{ Use of antibiotics } \\
\hline$\geq 3$ & $18(62.1 \%)$ & 17 (53.1\%) & 0.65 & 1.44 & $0.46-4.56$ \\
\hline Carbapenems & 12 (41.4\%) & 15 (46.9\%) & 0.86 & 0.80 & $0.26-2.49$ \\
\hline Vancomycin & $18(62.1 \%)$ & $13(40.6 \%)$ & 0.16 & 2.39 & $0.76-7.67$ \\
\hline $3^{\text {rd }}$ and $4^{\text {th }}$ generations Cef. ${ }^{¥}$ & 23 (79.3\%) & 23 (71.9\%) & 0.70 & 1.50 & $0.40-5.76$ \\
\hline \multicolumn{6}{|l|}{ Penicillins } \\
\hline Amphicilin & $1(3.4 \%)$ & $5(15.6 \%)$ & 0.20 & 0.19 & $0.01-1.92$ \\
\hline Oxacillin & $1(3.4 \%)$ & $10(31.2 \%)$ & $0.01^{\#}$ & 0.08 & $0.00-0.69$ \\
\hline Clindamicin & $8(27.6 \%)$ & $10(31.2 \%)$ & 0.97 & 0.84 & $0.24-2.90$ \\
\hline Quinolones & $10(34.5 \%)$ & $8(25.0 \%)$ & 0.59 & 1.58 & $0.46-5.52$ \\
\hline Others* & 11 (37.9\%) & $11(34.4 \%)$ & 0.98 & 1.17 & $0.36-3.78$ \\
\hline
\end{tabular}

Ä Mechanical ventilation, + Central vascular cateter, ++ Vesical Probe, +++ Nasogatric Probe, ¥ Cephalosporins, * Amicacin, Aztreonan, Metronidazol, Cetoconazol, Fluconazol, Nistatin, \# $\mathrm{p} \leq 0.05$.

having as etiological agents, more frequently, multiresistant bacteria such as ORSA $[15,28,29]$.

Infections by ORSA demand a more rigorous treatment evaluation, including choice of antibiotics, since they relate with greater morbidity and mortality, as compared to those caused by OSSA [8]. Metanalysis studies by Whitby, McLaws and Berry (2001) [30] and Cosgrove et al. (2002) [31], including results from various publications comparing the mortality risk among patients with bacteremia, those by ORSA presented increased mortality, when compared to OSSA-associated infections. Little data are available on VAPs, but Rello et al. (1994) [18] reported that the mortality rate directly related to VAPs by ORSA was significantly higher than that by OSSA. In our study, the mortality rate observed was also higher, but without statistical significance in the group with VAPs by ORSA (37.9\%) when compared to that of the group with OSSA (25\%).

The initial choice of the empirical antimicrobial scheme for the treatment of VAPs is of critical importance in determining a patient's clinical evolution, particularly against hospital mortality [32]. Early aggressive therapeutics and use of large-spectrum antibiotics, directed against more probable microorganisms, based on local vigilance data, is associated to reduced mortality rates [10,33-35]. Once the causal agent is defined, one must opt for the use of reduced spectrum antibiotics, compatible with the existing microorganism, i.e., treatment must be conducted based 
Table 2. Treatment and evolution of patients with ventilator-associated pneumonia (VAP) by S. aureus with resistance (ORSA) or sensitivity (OSSA) to oxacillin of the ICU for adult patients of the Uberlândia Federal University Hospital Clinic (HC-UFU), from May 2005 to April 2007.

\begin{tabular}{lcccc}
\hline Microorganism & $\begin{array}{c}\text { Adequate treatment } \\
\mathbf{N}\end{array}$ & $\begin{array}{c}\text { Mortality }^{\mathrm{e}} \\
\mathbf{N}=\mathbf{3 7}(\mathbf{1 3} \mathbf{( \% )}\end{array}$ & $\begin{array}{c}\text { Inadequate treatment }^{\mathrm{d}} \\
\mathbf{N}=\mathbf{2 4} \mathbf{( \% )}\end{array}$ & $\begin{array}{c}\text { Mortality }^{\mathrm{f}} \\
\mathbf{N}=\mathbf{6}(\mathbf{\%})\end{array}$ \\
\hline ORSA $^{\mathrm{a}}$ & $18(48.7 \%)$ & $7(53.8 \%)$ & $11(45.8 \%)$ & $4(66.7 \%)$ \\
OSSA $^{\mathrm{b}}$ & $19(51.3 \%)$ & $6(46.2 \%)$ & $13(54.2 \%)$ & $2(33.3 \%)$ \\
\hline
\end{tabular}

$* \mathrm{P}$ - a $\mathrm{x} \mathrm{b}=\mathrm{ns} ;$ c $\mathrm{x} \mathrm{d}=\mathrm{ns} ;$ e $\mathrm{x} \mathrm{f}=\mathrm{ns} . *$ Non-significant.

Figure 1. Etiological agents of patients with ventilatorassociated pneumonia (VAP) at the ICU for adult patients of the Uberlândia Federal University Hospital Clinic (HC-UFU), from May 2005 to April 2007.
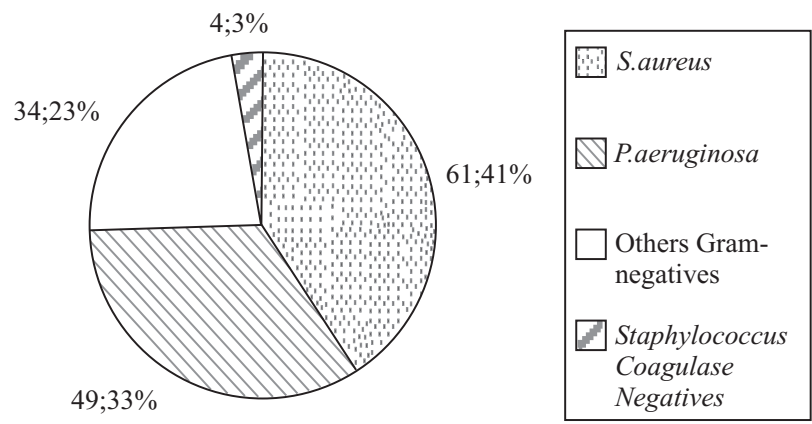

on the culture result, promoting de-escalating antibiotic strategy [10,34]. In our investigation, most patients (57.4\%, 35/ 61) were given three or more antibiotics empirically, without adopting the de-escalating antibiotic strategy after the microbiological results were provided. These conditions contribute to the emergence of antibiotics-resistant and multiresistant microorganisms reported by many authors $[8,15]$.

When empirical therapeutics is applied, particularly under the existing conditions in our ICU, there is a high likelihood of inadequate therapeutics to occur. The term inadequate antibiotic therapy is not standardized, but it is usually applied to define the conditions under which the initial use of antibiotics is inactive for the microorganism identified as etiological agent of the infection $[34,36]$. Although the definition is restricted to the spectrum of susceptibility to the antibiotics instead to a therapeutic clinical response, focusing only on microbiologically documented infections $[34,37]$ in the case of VAPs by OSSA, we have defined as inappropriate the use of vancomycin, because of its pharmacodynamic and pharmacokynetic characteristics related to the low levels reached in the epithelial lining fluid $[8,13]$, and the result obtained from its use versus that of penicillin isoxazolyl [38].Among our patients, the proportion of inadequate therapy was very high (39.3\%), the majority (54.2\%) in patients with VAP by OSSA. The inadequate use of antibiotics was identified as the most important risk factor for hospital mortality among 2000 critical VAP patients in general
Figure 2. Frequencies of $S$. aureus samples with sensitivity (OSSA) and resistance to oxacillin (ORSA), in the etiology of ventilator-associated pneumonia (VAP) at the ICU for adult patients of the Uberlândia Federal University Hospital Clinic (HC-UFU), from May 2005 to April 2007.

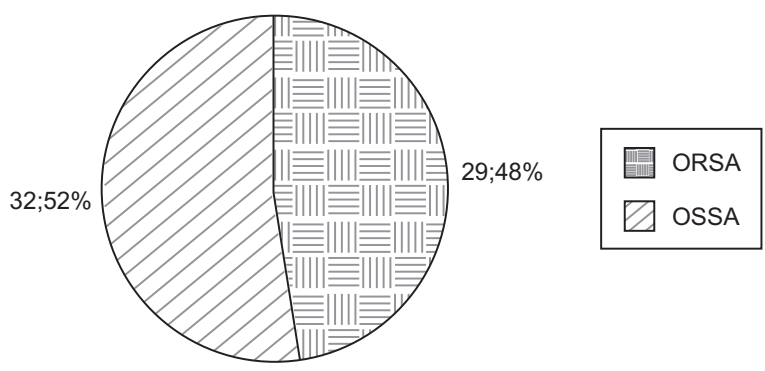

[36]. In our study, ORSA-infected patients had a similar mortality rate, regardless of whether the treatment was adequate (38.9\%) or inadequate (36.4\%), probably a reflection of older age and corticoid immune-suppression. Moreover, they are critical patients and other factors can be related with the mortality of the same ones. However, the mortality observed among patients with VAP by ORSA under inadequate treatment was double (66.7\%) that observed in pneumonias by OSSA (33.3\%), but without statistical significance, suggesting a bigger virulence of the phenotype with resistance to the oxacillin.

Gonzalez et al. (1999) [38] reported a relation between mortality and vancomycin treatment in patients with pneumonia by $S$. aureus, with high mortality rates in infections both by ORSA and OSSA, but extremely high $(50,0 \%)$ in those by ORSA, as observed in our series (53.8\%).

Superior clinical efficacy of linezolid over vancomycin in the treatment of VAPs by ORSA was reported by Wunderink et al. (2003) [39] and Kollef et al.(2004) [11] in retrospective doubleblind studies, with higher clinical survival and cure rates. In our hospital, vancomycin is still the choice antibiotic for therapeutics of infections by ORSA, since linezolid has not been standardized.

\section{Conclusions}

S. aureus is the main agent of VAPs in the ICU of the HCUFU with around half the cases by ORSA, associated with the development of late VAP, age over 60, use of corticoids and 
antibiotics, and a higher mortality (37.9\%) than that observed in the group by OSSA (25.0\%), ( $>0.05)$. Constant inadequate empirical therapeutics, including the use of vancomycin in VAPs by OSSA, was very high ( $40 \%)$ in the two groups, with a greater reflection on mortality by ORSA. At the moment, this antibiotic is the only option for pneumonias by ORSA in our hospital, characterized by a more somber prognosis.

\section{References}

1. Leroy O., Jaffré S., Escrivan T., et al. Risk Factors for Antimicrobial-Resistance Causative Pathogens in Critically Ill Patients. Chest 2003;123:2034-2042.

2. Alp E., Voss A. Ventilator Associated Pneumonia and Infection Control. Ann Clinic Microbiol Antimicrob 2006;5:7doi: 10.1186/ 1476-0711-5-7.

3. Park D.R. The Microbiology of Ventilator-Associated Pneumonia. Respirat Care 2005;50(6):742-65.

4. Pfaller M.A., Cormican M.G. Microbiology: The Role of the Clinical Laboratory. In: Wenzel R. P. Prevention and Control of Nosocomial Infections 3 ed. Baltimore: Williams \& Wilkins 1997.

5. Shorr A.F., Susla G.M., Kollef G.M. Linezolid for treatment of ventilator-associated pneumonia: a cost-effective alternative to vancomycin. Crit Care Med 2004; 32(1): 137-143.

6. Bergmans D.C.J.J., Bonten M.J.M. Methicillin-Resistant Staphylococcus aureus. In: Mayhall C. G. Hospital Epidemiology and Infection Control $3^{\text {a }}$ ed. Philadelphia: Lippincott Williams \& Wilkins, 2004.

7. Shurland S., Zhan M., Bradham D., Roghmann M. Comparison of Mortality Risk Associated With Bacteremia Due to MethicillinResistant and Methicillin-Susceptible Staphylococcus aureus. Infect Control and Hosp Epidemiol 2007;28(3):273-9.

8. Kollef M.H. Antibiotic management of ventilator-associated pneumonia due to antibiotic-resistant Gram-positive bacterial infection. Eur J Clin Microbiol Infect Dis 2005;24(12):794-803.

9. Ferrara A.M. Tratament of hospital-acquired pneumonia caused by methicillin-resistant Staphylococcus aureus. Int J Antimicrob Agents 2007;30(1):19-24.

10. Masterton R. The place of guidelines in hospital-acquired pneumonia. J Hosp Infect 2007;66:116-22.

11. Kollef M.H., Rello J., Cammarata S.K., et al. Clinical cure and survival in Gram-positive ventilator associated pneumonia: retrospective analysis of two double-blind studies comparing linezolid with vancomycin. Intensive Care Med 2004;30:388-94.

12. Cruciani M., Gatti G., Lazzarini L., et al. Penetration of vancomycin into human lung tissue. J Antimicrob Chemother 1996; $35: 865-71$.

13. Lamer C., De Beco V., Soler P., et al.; Analysis of vancomycin entry into pulmonary lining fluid by bronchoalveolar lavage in critically ill patients. Antimicrob Agents Chemother 1993;37:281-6.

14. Conte J., Golden J., Kipps J., Zurlinden E. Intrapulmonary pharmacokinetics of linezolid. Antimicrob Agents Chemother 2002; $46: 1475-80$.

15. Trouillet J.L., Chastre J., Vuagnat A., et al. Ventilator-Associated Pneumonia Caused by Potentially Drug-resistat Bacteria. Am J Respir Crit Care Méd 1998;157(2):531-9.

16. Dean A.G. Epi Info, Versão 5.0: A word processing, database, and statistics program for epidemiology on microcomputers. Stone Montain, G.A:USD, Ins; 1995.

17. Diaz O., Diaz E., Rello J. Risk factors for pneumonia in the intubated patient. Infect Dis Clin North America 2003;17:697-705.

18. Rello J., Torres A., Ricart M., et al. Ventilator-associated pneumonia by Staphylococcus aureus: comparison of methicillin-resistant and methicillin-sensitive episodes. Am J Respir Crit Care Med 1994;150(6 Pt 1):1545-9.
19. Vincent J.L. Nosocomial infectious in adult intensive-care units. Lancet 2003;361:2068-77.

20. National Nosocomial Infections Surveillance (NNIS) system report, data summary from January 1992-June 2001, issued August 2001. Am J Infect Control 2001;29:404-21.

21. Teixeira P.J.Z., Hertz F.T., Cruz D.B., et al. Pneumonia associada à ventilação mecânica: impacto da multirresistência bacteriana na morbidade e mortalidade. J Bras Pneumol 2004;30(6):540-8.

22. Carrilho C.M.D.M. Fatores associados ao risco de desenvolvimento de pneumonia hospitalar na Unidade de Terapia Intensiva do Hospital Universitário Regional do Norte de Paraná. Rev Bras Med Trop 1999;32:455-6.

23. Woodford N. Biological counterstrike: antibiotic resistance mechanisms of Gram-positive cocci. Clin Microbial Infect 2005;11(suppl.3):2-21.

24. Martins A., Cunha M.L.R.S. Methicillin Resistance in Staphylococcus aureus and Coagulase-Negative Staphylococci: Epidemiological and Molecular Aspects. Microbiol Immunol 2007;51(9):787-95.

25. Cunha B.A. A useful clinical approach to community-acquired methicillin-resistant Staphylococcus aureus (CA-MRSA) infections. J Hosp Infect 2008;68:271-3.

26. Lam A.P., Wunderink R.G. Methicillin-Resistant Staphylococcus aureus Ventilator-Associated Pneumonia: Strategies to Prevent and Treat. Semin Respir Crit Care Med 2006;27(1):92-103.

27. Shlaes D.M., Gerding D.N., John Jr J.F. et al. Society for Healthcare Epidemiology of America and Infectious Diseases Society of America Joint Committee on the Prevention of Antimicrobial Resistance: guedelines for the prevention of antimicrobial resistance in hospitals. Infect Control Hosp Epidemiol 1997;18(4):275-91.

28. Kollef M.H. The importance of antimicrobial resistance in hospital-acquired and ventilator-associated pneumonia. Cur Anaesth Crit Care 2005;16:209-19.

29. Chastre J., Fagon, J. Ventilator-associated Pneumonia. Am J Respir Crit Care Med 2002;165:867-903.

30. Whitby M., Mclaws M.L., Berry G. Risk of death from methicillinresistant Staphylococcus aureus bacteraemia: a meta-analysis. Med J Aust 2001;175:264-7.

31. Cosgrove S.E., Sakoulas G., Perencevich E.N., et al. Comparison of mortality related to meticillin-resistant and meticillinsusceptible Staphylococcus aureus bacteremia: a meta-analysis. Clin Infect Dis 2002;36:53-9.

32. Kollef M.H., Morrow L.E., Niederman M.S., et al. Clinical characteristics and treatment patterns among patients with ventilator-associated pneumonia. Chest 2006;129(5):1210-8.

33. Rello J., Gallego M., Mariscal D., et al. The Value of Routine Microbial investigation in Ventilator-Associated Pneumonia. Am J Respir Crit Care Med 1997;156:196-200.

34. Höffken G., Niederman M.S. The Importance of a De-escalating Strategy for Antibiotic Treatment of Pneumonia in the ICU. Chest 2002;122:2183-96.

35. Luna C.M., Nujacich P., Niederman M.S., et al. Impact of BAL data on the therapy and outcome of ventilator-associated pneumonia. Chest 1997;111:676-83.

36. Kollef M.H., Sherman G., Ward S., et al. Inadequate antimicrobial treatment of infections: a risk factor for hospital mortality among critically ill patients. Chest 1999;115:462-74.

37. Wunderink R.G. Mortality and ventilator-associated pneumonia: the best antibiotics may be the least antibiotics [editorial]. Chest 1993;104:993-5.

38. Gonzalez C., Rubio M., Romero-Vivas J., et al. Bacteremic pneumonia due to Staphylococcus aureus: a comparison of disease caused by methicillin-resistant and methicillin susceptible organisms. Clin Infect Dis 1999;29:1171-7.

39. Wunderink R.G., Rello J., Cammarata S.K., et al. Linezolid vs. vancomycin: analysis of double-blind studies of patients with methicillin-resistant Staphylococcus aureus nosocomial pneumonia. Chest 2003;124:1789-97. 